\title{
The Use of Magnetic Resonance Imaging for the Evaluation of the Porcine Intervertebral Disc Condition Under Treatment
}

\author{
Seweryn Lipiński ${ }^{1}$ - Katarzyna Jezierska-Woźniak ${ }^{2,3} \cdot$ Aleksandra Habich $^{2,3}$. \\ Joanna Wojtkiewicz ${ }^{4}$ Kamila Milewska ${ }^{2}$. Piotr Holak ${ }^{5}$. \\ Wojciech Maksymowicz ${ }^{2} \cdot$ Monika Barczewska²
}

Received: 6 April 2019 / Revised: 17 June 2019 / Published online: 2 July 2019 (C) The Author(s) 2019

\begin{abstract}
One of the methods of evaluation of degenerative disc disease therapies' effectiveness is the use of animal models and magnetic resonance imaging (MRI), for the evaluation of the degree of damage and possible subsequent regeneration of intervertebral discs (IVDs). The purpose of our study was to propose the methodology that can be used for assessing the condition of the porcine IVDs based on scans obtained in MRI study. Our research is based on the MRI scans obtained for the purpose of evaluation of the process of IVDs regeneration after administration of mesenchymal stem cells isolated from bone marrow after the damage caused by the surgical laser vaporization. Proposed method of segmentation utilizes basic methods of digital image processing implemented with the use of Matlab environment. The outcome of the described method is binary mask giving direct information about locations of IVDs in MRI scans. We present the results of the IVD condition evaluation with the use of described method. We also present differences that may appear in the assessment of the same cases based on different cross-sections of the same MRI research. (1) The use of coronal imaging plane is recommended to use, as it can be indicated as more reliable than sagittal. (2) Use of the basic geometrical shapes as the discs' shape approximation can lead to significant errors in the evaluation of the experiment results - the borders of IVDs should be determined using more complex shapes, i.e. considering the anatomy.
\end{abstract}

Seweryn Lipiński

seweryn.lipinski@uwm.edu.pl

Extended author information available on the last page of the article 


\section{Introduction}

Intervertebral Disc (IVD) is a part of a spine that is interposed between two adjacent vertebral bodies. IVDs are deformable structures built by fibrocartilage-each of them acts as a buffer, allowing movements of the vertebrae and acting as a ligament holding the vertebrae together. Diseases of the IVDs, also known as discopathies, are one of the most common civilization diseases [1-3]. Degenerative disc disease (DDD) is initiated through biochemical changes in the structure of discs [4-6]. It is responsible for the pain syndrome and in some cases the weakness of extremities [7, 8].

There are many studies concerning methods of discopathy treatment [6, 9-13]. New experimental therapies aim to treat DDD at the genetic, cellular, and molecular levels. Cellular therapies may stop or reduce the pain associated with the degeneration of IVDs. The aim of this therapy is the restoration of intercellular matrix and restoring the biochemical function of discs. Recent preclinical studies indicate that mesenchymal stem cells have the ability to repair damaged discs by differentiating toward the nucleus pulposus-like cells [14, 15].

Animal models, in which in a certain way is initiated the process of degeneration of the IVDs, are often used to evaluate the effectiveness of particular therapies. Many of them base on animal models, like dogs [16], mice [17], pigs [9, 18], goats [19], rabbits [20] or rats [21]. Most commonly used method of determination of the degree of degeneration/damage and possible subsequent regeneration of IVDs is magnetic resonance imaging (MRI) [9, 17, 22-25]-mainly because this imaging technique is safe and non-invasive. Quantification of temporal changes in disc degeneration is performed based on the MRI signal intensity inside IVDs, i.e. discs cross-sections [9, 26-28]. While using this approach there appear factors, whose impact is often underestimated.

First of them are differences that may arise in the assessment of the same cases based on images obtained in different (i.e. coronal or sagittal) cross-sections of the same MRI research.

The second problem is IVD segmentation. As the manual disc segmentation is time-consuming and the influence of human perception is always hard to assess, there is a need of automatic segmentation of IVD area from the MRI scans. Another problem is that severely damaged discs can have unclear boundaries, very hard to delineate.

Both these problems were the inspiration for this paper, which is the presentation of our effort to evaluate properly the condition of the porcine IVDs based on MRI. We propose a method of automatic segmentation of IVDs from MRI scans, basing on some specific properties of spine MRI. It is worth mentioning that the method described in this paper was successfully utilized in Ref. [9].

It should be also noted that there are studies concerning IVD segmentation [29-33]. Our method has slightly different aim. It is destined for the purpose of preliminary segmentation of IVDs in an undamaged spine and next using it for segmentation of degenerated discs in the same subject. There are two main advantages of proposed method. The first of them is its simplicity, which is achieved due to the uncomplicated binarization algorithm, often embedded in computing 
environments, (Otsu method of automatic thresholding). The second advantage results from the use of basic operators of morphological image processing, which are easily programmable.

\section{Materials}

\subsection{Animals and Surgical Procedures}

The surgical procedure described previously was followed in this study [34].

All animals were housed and treated in accordance with rules approved by the ethics committee (conforming to Principles of Laboratory Animal Care, NIH publication no. 86-23, revised 1985).

\subsection{Isolation of MSCs}

MSCs were isolated from the bone marrow of porcine donors according to their adhesive properties to tissue culture plastic under sterile conditions. Briefly, a phosphate-buffered saline (PBS)-diluted cell fraction of bone marrow was layered over a Ficoll density gradient $(1.077 \mathrm{~g} / \mathrm{mL}$, GE Healthcare), followed by centrifugation at $400 \mathrm{~g}$ at room temperature for $40 \mathrm{~min}$. Nucleated cells were collected, diluted with two volumes of PBS, centrifuged twice at $100 \mathrm{~g}$ for $10 \mathrm{~min}$, and finally resuspended in culture medium. Cells were plated and expanded in a T-75 flask and grown for 14 days at $37{ }^{\circ} \mathrm{C}$ and $5 \% \mathrm{CO}_{2}$, with medium changes every 3 days.

\subsection{IVD Vaporization Procedure}

The IVD vaporization procedure was performed using the transcutaneous needle insertion under fluoroscopy control and the energy of the medical laser, as previously described [34].

\subsection{Cell Transplantation}

Immediately prior to transplantation, cells were washed with PBS, harvested by trypsinization, and suspended in HyStem hydrogel (Glycosan Biosystems) at a concentration of $1 \times 106 / \mathrm{mL}$. Autogenic MSCs embedded in HyStem hydrogel were transplanted into the degeneration-induced discs in anesthetized pigs. The entire cell transplantation procedure was performed as described previously [34].

\subsection{Magnetic Resonance Imaging}

Our research is based on the MRI study obtained for the purpose of evaluation of the process of regeneration of IVDs after administration of mesenchymal stem cells isolated from bone marrow in porcine animal model, after the damage caused by the surgical laser vaporization of the nucleus pulposus [9]. MRI scans 
of the pigs were obtained using a 3 T MRI scanner (Magnetom TRIO-Siemens production). The pigs were examined in a supine position and the T2-weighted images of the spine were acquired with the same coil geometry. A spinal surface coil was placed over the backs of the animals. Parameters of imaging were as follows: $\mathrm{TE} / \mathrm{TR}=102 / 4500, \mathrm{AV}=1$, Res $=560 \times 560 \times 3000 \mu \mathrm{m}$. MRI scans were obtained for the time of 4,8 , and 12 weeks from the time of damage and at the start of the experiment.

Exemplary images from the MRI study used in the article are shown in Fig. 1. Figure 1a presents a view of a swine spine in a sagittal plane while Fig. 1b presents a view of the same spine in a coronal (frontal) plane. Figure 1c-e presents MRI scan of the same pig after 4,8 and 12 weeks from induction of disc

A

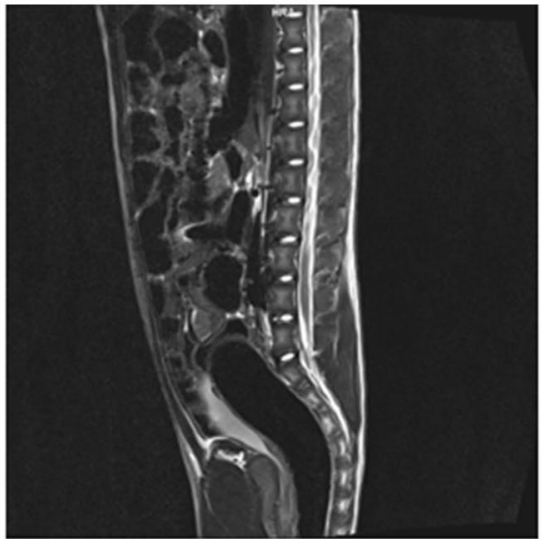

C

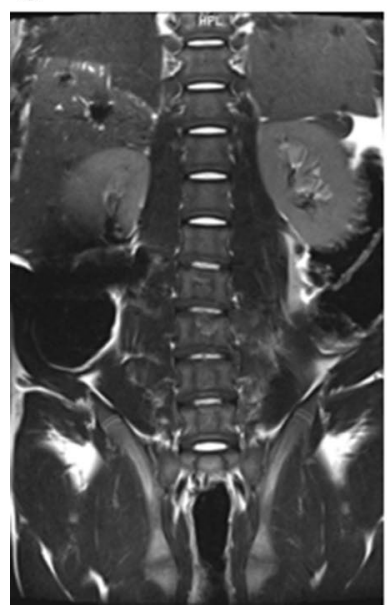

B

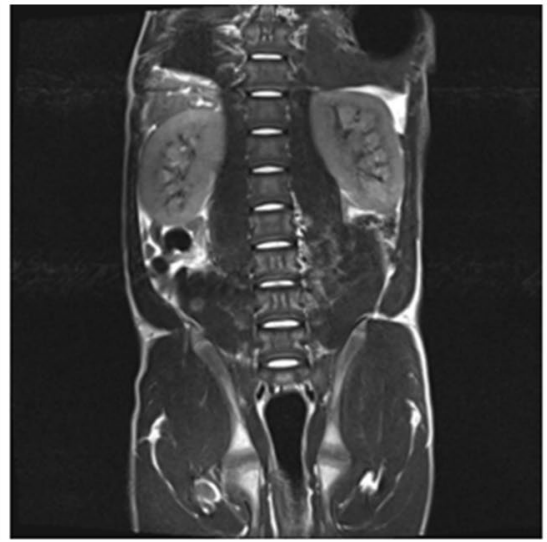

$\mathbf{E}$

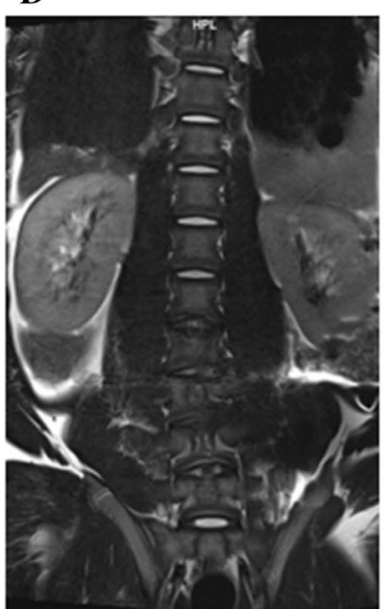

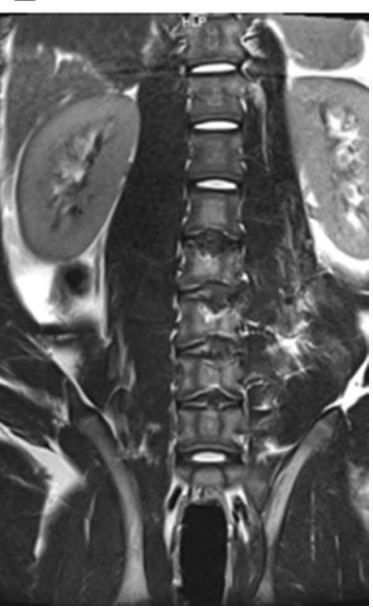

Fig. 1 Exemplary MRI images of a pig spine in a sagittal (a) and coronal (b) plane at the start of the experiment and a scan of the same animal after 4, 8 and 12 weeks after exposure to the laser surgery (c, $\mathbf{d}$ and $\mathbf{e}$, respectively, the coronal plane) 
degeneration. It is easy to notice that borders of IVDs after induction of IVDs degeneration are much harder to delineate.

\section{Methods}

\subsection{Imaging Plane}

The evaluation of the reliability of assessment of IVD degeneration depending on the choice of imaging plane in MRI study was performed based on the manually outlined contours of discs. On that basis, degeneration of IVD was assessed through changes in the intensity of the MRI signal in time of 4, 8 and 12 weeks after surgery-the data for the graph were obtained from both (i.e. coronal and sagittal) imaging planes. MRI signal intensity was normalized by the MRI signal for the reference discs, i.e. healthy IVDs of the same animal.

\subsection{Methods of Image Processing Used in Intervertebral Disc Segmentation}

\subsubsection{Binarization}

The method of segmentation proposed in the paper utilizes the two basic morphological operations: erosion and dilation, more precisely their compositions, i.e. opening and closing. The operators of morphological processing are particularly useful for the analysis of binary images, so the MRI images need to be previously binarized. The original eight-bit 'grayscale' MRI images have to be converted into one-bit 'binary' images. To discriminate the greyscale images, i.e. to convert them into binary matrices, the Otsu method of automatic global thresholding is used. This method is one of the most referenced methods $[35,36]$, often embedded in computing environments. It selects the binarization threshold by minimizing the weighted sum of within-class variances of the foreground and background pixels $[35,36]$. Binarized image from Fig. $1 \mathrm{~b}$ is shown in Fig. 2.

Figure 2 shows that in a binarized image, there are clearly noticeable shapes of IVDs, but there are many other undesirable white regions as well. In order to remove them, we utilize operators of mathematical morphology applied in image processing.

\subsubsection{Morphological Image Processing}

The methods of morphological image processing can be successfully implemented for the purpose of medical image segmentation [37-41]. The operators of mathematical morphology take two items of data as input: the image to be processed and a structuring element that is a matrix used to define the neighborhood size and shape. The two fundamental operators of mathematical morphology applied in image processing are dilation and erosion. The erosion of the binary image $B$ by the structuring element $S$ is defined as follows $[42,43]$ :

$$
B \otimes S=\left\{x, y: S_{x, y} \subseteq B\right\}
$$


Fig. 2 Image from Fig. 1b after binarization using Otsu method of automatic thresholding

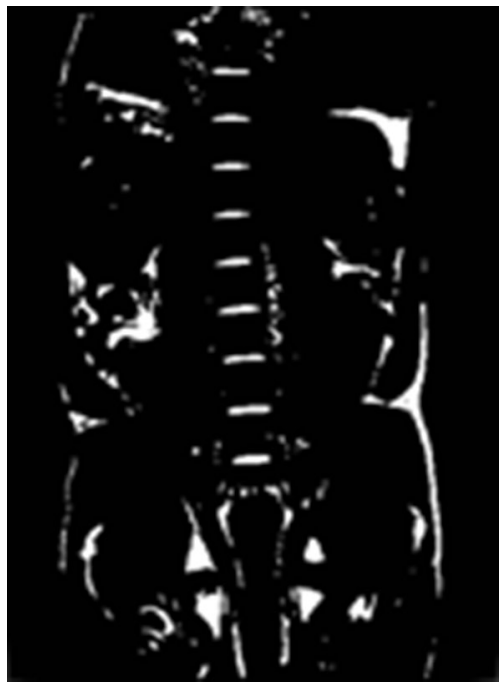

The basic effect of the erosion operation on a binary image is to erode the boundaries of the foreground pixel region.

The dilation of the binary image $B$ by the structuring element $S$ is defined by the following formula $[42,43]$ :

$$
B \oplus S=\left\{x, y: S_{x, y} \cap B \neq \emptyset\right\} .
$$

The basic effect of the dilation on a binary image is to enlarge the boundaries of the foreground pixel region.

The operations of morphological closing $B \bullet S$ and morphological opening $B \circ S$ in binary and grayscale images are the combinations of erosion and dilation, both using the same structuring element. Morphological opening is erosion followed by dilation and morphological closing is dilation followed by erosion. Main features of these operations are $[42,43]$ the following:

- neither of the operations changes shape or size of big figures with smoothed edges;

- opening removes small objects and details from an image; it can also uncouple objects with thin connections;

- closing fills in waists and gaps inside a processed figure; it can also join objects that are close to each other.

The sensitivity of the morphological operations to specific shapes appearing in the processed image is dependent on the size and shape of the structuring element. The proposed method uses this feature to remove unnecessary objects from the previously binarized image (i.e. image shown in Fig. 3). Expected results depend on an adequate choice of a structuring element as well as on its shape.

To remove the leftover pixels, we use the following, experimentally designed, morphological operator: 
Fig. 3 Binary mask obtained for the MRI scan from Fig. 1b (a) and intervertebral discs separated with the use of this mask (b)

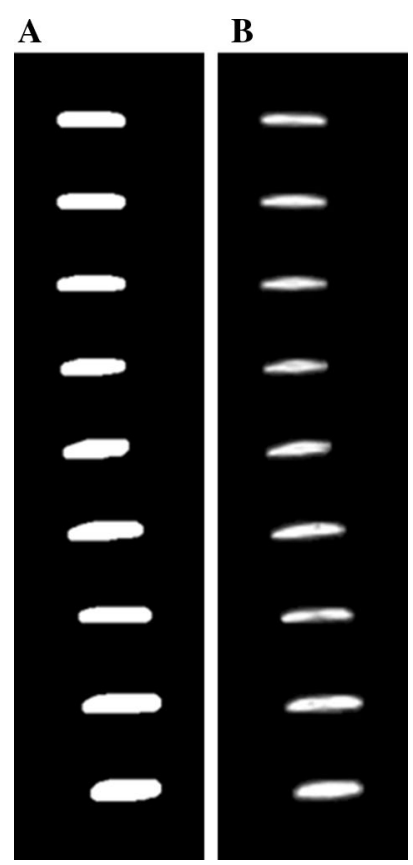

$$
\mathrm{MO}=\left(B \circ S_{1}\right) \oplus S_{2},
$$

where $S_{1}$ and $S_{2}$ are structuring elements with following shapes and sizes: rectangle with dimensions of 4 by 30 pixels and disc with a radius equal to 4 pixels $\left(S_{1}\right.$ and $S_{2}$, respectively).

As the result of performed morphological operation, all the undesired objects are removed from the background. Figure 3a shows the cleared image from Fig. 2. This image represents a mask that gives direct information of which pixels of image from Fig. 1b should be treated as belonging to the area representing IVDs. Figure $3 \mathrm{~b}$ shows IVDs segmented from Fig. $1 \mathrm{~b}$ with the use of mask from Fig. 3a.

\subsubsection{Segmentation of Images Containing Degenerated Intervertebral Discs}

As mentioned above, degenerated IVDs can have boundaries that are very hard to delineate. It is clearly noticeable in Fig. 1c, d and especially e, which shows the scans of the pig spine after 4, 8 and 12 weeks from the surgery. Even a superficial assessment of these images allows noticing that with the progress of damage, problems with the delimitation of the IVDs on MRI scans may appear. We propose to use the mask obtained for non-degenerated discs and then to superimpose it on the MRI scans of degenerated IVDs with the use of position of non-impacted IVDs as reference points in the process of matching mask to the images obtained in the subsequent MRI studies. 


\section{Results and Discussion}

\subsection{Influence of Imaging Plane Choice}

There are studies, where MRI scans in the sagittal plane of a spine (shown in Fig. 1a) are being used for the purpose of the evaluation of degeneration/regeneration of IVDs [19, 44]. In our opinion, this approach may result in less reliable assessment than one based on the coronal (frontal) plane (shown in Fig. 1b). This statement stems directly from the fact that the frontal plane provides more information. In case of comparison of images shown in Fig. 1a, b, the difference is as high as $155 \%$ in favour of the frontal plane (4153 vs. 1626 pixels covering the area of interest, i.e. IVDs). Figure 4 shows (on an example of one swine) the degeneration of IVD assessed through changes in the intensity of the MRI signal in time of 4, 8 and 12 weeks after surgery - the data for the graph were obtained from both imaging planes.

As it can be seen, data obtained for two imaging planes show a similar pattern of changes in damage over time. However, the data obtained for 8 week from the surgery shows difference, as the evaluation in the frontal plane indicates less damage. Given the fact that in that plane about 2.5-times more the measurement data were available, it seems reasonable to say that it is more reliable.

Summarizing, in our opinion approach using sagittal plane is not recommended, as it carries less information that coronal (frontal) one.

At this point it is worth noting that axial sequences can be also used when assessing porcine IVDs condition [45, 46]. This approach, although it can be very useful in case of classic diagnosis, cannot be used for the purpose of objective (i.e. given numerically) evaluation of the discs condition under treatment, as in that case it is impossible to obtain exactly the same cross-section of the IVD, what in turn makes direct comparison impossible. The same comment refers to 3D images.

\subsection{Segmentation of Intervertebral Discs}

The effect of procedure of segmentation of IVDs that is described in subsection 3.2 is shown in Fig. 5 on one undamaged and four degenerating discs (Fig. 1b-e).

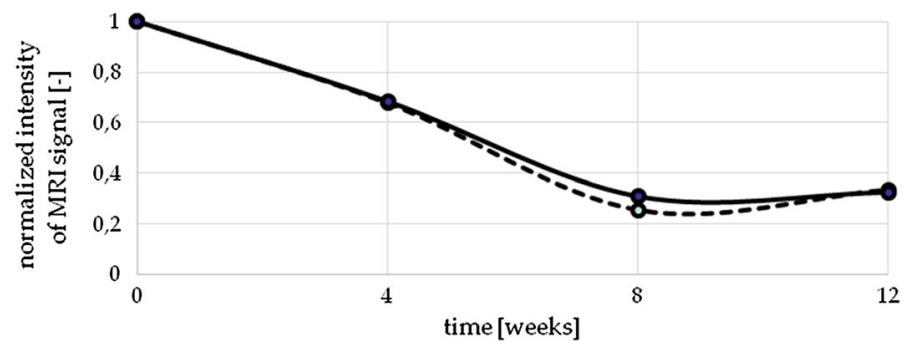

Fig. 4 Progress of the degeneration of the intervertebral discs evaluated in the coronal (solid line) and the sagittal plane (dashed line) 


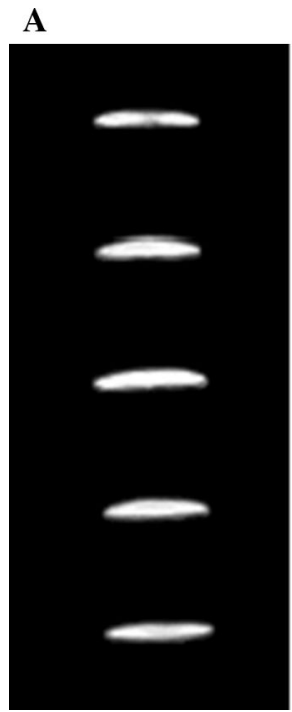

\section{B}

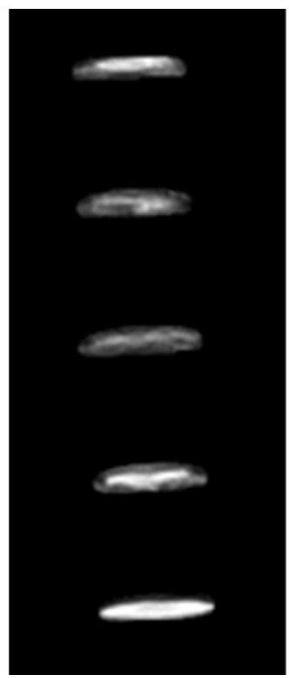

C

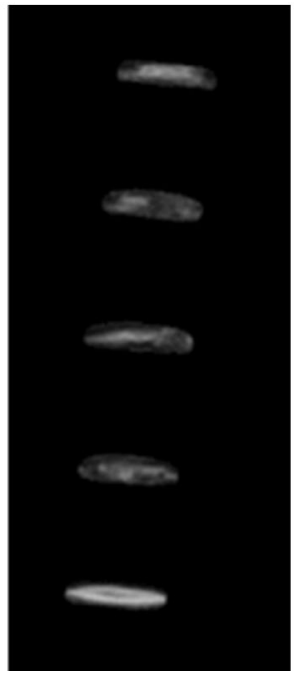

D

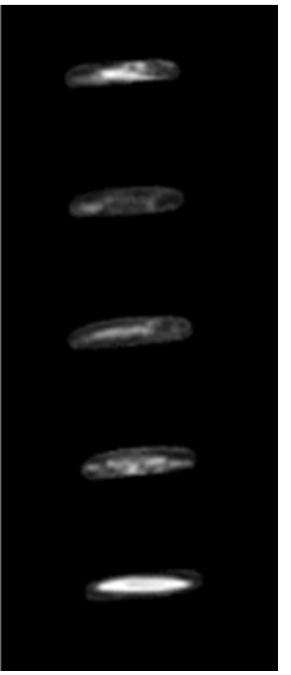

Fig. 5 Process of four IVDs' degeneration-MRI scans obtained on the start of the experiment (a), and after 4 (b), 8 (c) and 12 weeks (d); the 5th disc from the top is undamaged disc, next used for normalization of the MRI signal intensity value

Values of MRI signal intensity for four IVDs of the above exemplary pig spine are shown in Fig. 6. Data used for creating this image was obtained using the described method. In order to normalize the MRI signal intensity, it was calculated through dividing the signal intensity of the impacted disc by that of the normal disc of the same pig.

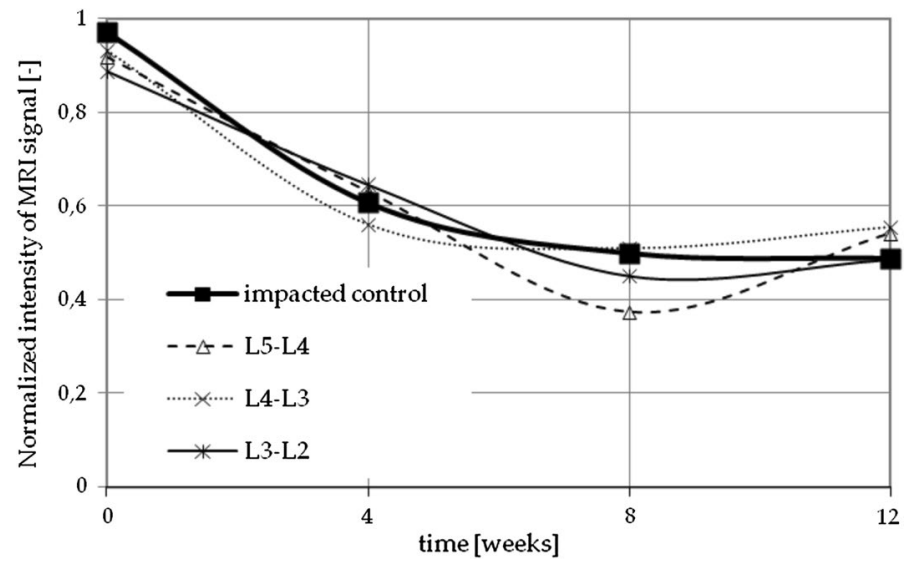

Fig. 6 Temporal changes in intervertebral disc degeneration observed as MRI signal intensity: thick line represents IVD used as impacted control, thin lines represent three damaged IVDs after mesenchymal stem cells transplantation 
In the context of IVD segmentation, the most common approach is the one in which the human operator manually indicates an elliptical or a rectangular area of the disc in the MRI scan. On the one hand, this approach allows avoiding an extremely time-consuming process of manual delimitation of an area of interest; on the other hand, it is difficult to treat this approach as sufficiently reliable. Figure 7 shows the progress of the degeneration of the IVDs evaluated based on the area of interest indicated manually, as a rectangular area, as well as using the proposed method. There are clearly noticeable differences in assessing the degree of degeneration of the discs. The differences at such level cannot be neglected, as they can lead to misinterpretation of the results of the experiment. For this reason, we recommend the use of methods of (semi)automatic segmentation area of IVDs, such as the one proposed in the paper.

\section{Conclusions}

Our study attempted to identify the most reliable methodology that could be used to assess the degree of degeneration/regeneration of IVDs. The paper presents inter alia a methodology of assessing the degree of degeneration of the porcine IVDs based on MRI. We propose a procedure basing on some specific properties of MRI images of porcine spine, specifically the fact that IVDs are visible in these scans in similar way in terms of their contour and size.

An advantage of the proposed method is its simplicity, achieved due to the uncomplicated, often embedded in computing environments binarization algorithm, and next basic operators of morphological image processing, which are also easily programmable.

The outcome is binary mask giving direct information about locations of IVDs in MRI scans. The obtained mask is next being used to separate locations of IVDs in following MRI studies of the same animals, in cases where they are much harder to delineate due to their degeneration.

Other conclusions that can be drawn based on our study are as follows:

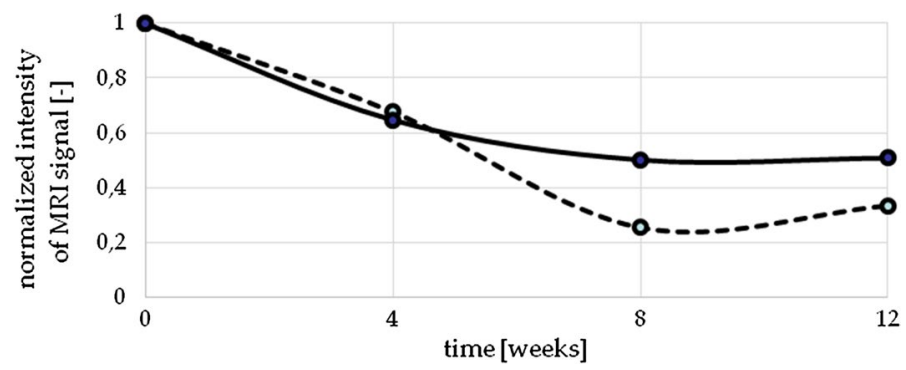

Fig. 7 Progress of the degeneration of the intervertebral discs evaluated in the coronal (frontal) plane (Fig. 1b-e), based on the area of interest referred to as the rectangular (dashed line) and with the use of the described method (solid line) 
Due to the greater number of available measurement data, the use of frontal (coronal) plane is recommended, as it can be indicated as more reliable than sagittal.

Use of the one of the basic geometrical shapes as the discs' shape approximation can lead to significant errors in the evaluation of the results of the experiment- the borders of the IVD should be determined using more complex shapes, i.e. considering the anatomy. Advisable is the use of one of the methods of automatic or semimanual segmentation of the area of interest.

Funding This study was funded by National Science Center (NCN) Grants number 2012/07/B/ NZ4/01427, and 2012/05/D/NZ3/02028. The funders had no role in study design, data collection and analysis, decision to publish, or preparation of the manuscript.

\section{Compliance with Ethical Standards}

Conflict of interest The authors declare no conflict of interest.

Ethical approval All applicable international, national, and/or institutional guidelines for the care and use of animals were followed.

Open Access This article is distributed under the terms of the Creative Commons Attribution 4.0 International License (http://creativecommons.org/licenses/by/4.0/), which permits unrestricted use, distribution, and reproduction in any medium, provided you give appropriate credit to the original author(s) and the source, provide a link to the Creative Commons license, and indicate if changes were made.

\section{References}

1. G.B. Andersson, Lancet 354, 581-585 (1999). https://doi.org/10.1016/S0140-6736(99)01312-4

2. R.A. Deyo, D. Cherkin, D. Conrad, E. Volinn, Ann. Rev. Public Health 12, 141-156 (1991). https:// doi.org/10.1146/annurev.pu.12.050191.001041

3. K. Koszela, S. Krukowska, M. Woldańska-Okońska, Pediatr. Med. Rodz. 13(3), 344-351 (2017). https://doi.org/10.15557/pimr.2017.0036

4. J.A. Buckwalter, Spine 20(11), 1307-1314 (1995)

5. N.V. Vo, R.A. Hartman, P.R. Patil, M.V. Risbud, D. Kletsas, J.C. Iatridis, J.A. Hoyland, C.L. Le Maitre, G.A. Sowa, J.D. Kang, J. Orthop. Res. 34, 1289-1306 (2016). https://doi.org/10.1002/ jor.23195

6. J.P. Urban, S. Robert, Arthritis Res. 5, 120-130 (2003). https://doi.org/10.1186/ar629

7. K. Luoma, H. Riihimaki, R. Luukkonen, R. Raininko, E. Viikari-Juntura, A. Lamminen, Spine 25, 487-492 (2000)

8. F.M. Phillips, P.J. Slosar, J.A. Youssef, G. Andersson, F. Papatheofanis, Spine 38(7), E409-E422 (2013). https://doi.org/10.1097/BRS.0b013e3182877f11

9. M. Barczewska, K. Jezierska-Woźniak, A. Habich, S. Lipiński, P. Holak, W. Maksymowicz, J. Wojtkiewicz, Folia Neuropathol. 56(2), 124-132 (2018). https://doi.org/10.5114/fn.2018.76616

10. I. Boyraz, A. Yildiz, B. Koc, H. Sarman, BioMed. Res. Int. (2015). https://doi. org/10.1155/2015/304328 (article ID 304328)

11. A.J. Freemont, A. Watkins, C. Le Maitre, M. Jeziorska, J.A. Hoyland, J. Pathol. 196, 374-379 (2002). https://doi.org/10.1002/path.1050

12. C. Nguyen, I. Boutron, G. Baron, K. Sanchez, C. Palazzo, R. Benchimol, G. Paris, É. James-Belin, M.M. Lefèvre-Colau, J. Beaudreuil, J.D. Laredo, A. Béra-Louville, A. Cotton, J.L. Drapé, A. 
Feydy, P. Ravaud, F. Rannou, S. Poiraudeau, Ann. Int. Med. 166(8), 547-556 (2017). https://doi. org/10.7326/M16-1700

13. D. Sakai, Eur. Spine J. 17, 452-458 (2008). https://doi.org/10.1007/s00586-008-0743-5

14. G. Vadala, F. Russo, L. Ambrosio, M. Loppini, V. Denaro, World J. Stem Cells 8, 185-201 (2016). https://doi.org/10.4252/wjsc.v8.i5.185

15. G. Vadala, F. Russo, A. Di Martino, V. Denaro, J. Tissue Eng. Regen. Med. 9, 679-690 (2015). https ://doi.org/10.1002/term.1719

16. K. Serigano, D. Sakai, A. Hiyama, F. Tamura, M. Tanaka, J. Mochida, J. Orthop. Res. 28, 12671275 (2010). https://doi.org/10.1002/jor.21147

17. F. Rannou, T.-S. Lee, R.-H. Zhou, J. Chin, J.C. Lotz, M.-A. Mayoux-Benhamou, J.P. Barbet, A. Chevrot, J.Y.-J. Shyy, Am. J. Pathol. 164, 915-924 (2004). https://doi.org/10.1016/S0002 $-9440(10) 63179-3$

18. M. Rubessa, K. Polkoff, M. Bionaz, E. Monaco, D.J. Milner, S.J. Holllister, M.S. Goldwasser, M.B. Wheeler, Anim. Biotechnol. 28(4), 275-287 (2017). https://doi.org/10.1080/10495398.2017.12791 69

19. Y. Zhang, S. Drapeau, S.A. Howard, E.J.M.A. Thonar, D.G. Anderson, Spine 36, 372-377 (2011). https://doi.org/10.1097/BRS.0b013e3181d10401

20. S. Sobajima, A.L. Shimer, R.C. Chadderdon, J.F. Kompel, J.S. Kim, L.G. Gilbertson, J.D. Kang, Spine J. 5, 14-23 (2005). https://doi.org/10.1016/j.spinee.2004.05.251

21. A.C. Issy, V. Castania, M. Castania, C.E.G. Salmon, M.H. Nogueira-Barbos, E.D. Bel, H.L.A. Defino, Braz. J. Med. Biol. Res. 46, 235-244 (2013). https://doi.org/10.1590/1414-431X20122429

22. M. Matsumoto, Y. Fujimura, N. Suzuki, Y. Nishi, M. Nakamura, Y. Yabe, H. Shiga, J. Bone Jt. Surg. Br. 80, 19-24 (1998)

23. C.M. Jeng, T.C. Cheng, C.H. Kung, H.C. Hsu, Eur. Spine J. 20(3), 408-413 (2011). https://doi. org/10.1007/s00586-010-1547-y

24. M.G. Muriuki, R.M. Havey, L.I. Voronov, G. Carandang, M.R. Zindrick, M.A. Lorenz, L. Lomasney, A.G. Patwardhan, J. Orthop. Res. 34(8), 1389-1398 (2016). https://doi.org/10.1002/jor.23232

25. C.W. Pfirrmann, A. Metzdorf, M. Zanetti, J. Hodler, N. Boos, Spine 26(17), 1873-1878 (2001)

26. T. Kanda, M. Osawa, H. Oba, K. Toyoda, J.I. Kotoku, T. Haruyama, K. Takeshita, S. Furui, Radiology 275(3), 803-809 (2015). https://doi.org/10.1148/radiol.14140364

27. D. Sakai, J. Mochida, T. Iwashina, A. Hiyama, H. Omi, M. Imai, T. Nakai, K. Ando, T. Hotta, Biomaterials 27(3), 335-345 (2006). https://doi.org/10.1016/j.biomaterials.2005.06.038

28. V.V. Upasani, C.L. Farnsworth, R.C. Chambers, T.P. Bastrom, G.M. Williams, R.L. Sah, K. Masuda, P.O. Newton, J. Bone Jt. Surg. 93, 1408-1416 (2011)

29. C. Chen, D. Belavy, W. Yu, C. Chu, G. Armbrecht, M. Bansmann, D. Felsenberg, G. Zheng, IEEE Trans. Med. Imaging 34(8), 1719-1729 (2015). https://doi.org/10.1109/TMI.2015.2403285

30. M.W. Law, K. Tay, A. Leung, G.J. Garvin, S. Li, Med. Image Anal. 17(1), 43-61 (2013). https://doi. org/10.1016/j.media.2012.06.006

31. S.K. Michopoulou, L. Costaridou, E. Panagiotopoulos, R. Speller, G. Panayiotakis, A. ToddPokropek, IEEE Trans. Biomed. Eng. 56(9), 2225-2231 (2009). https://doi.org/10.1109/ TBME.2009.2019765

32. S. Ruiz-España, E. Arana, D. Moratal, Comp. Biol. Med. 62, 196-205 (2015). https://doi. org/10.1016/j.compbiomed.2015.04.028

33. A. Wong, A. Mishra, J. Yates, P. Fieguth, D.A. Clausi, J.P. Callaghan, IEEE Trans. Biomed. Eng. 56(11), 2748-2751 (2009). https://doi.org/10.1109/TBME.2009.2027225

34. M. Barczewska, J. Wojtkiewicz, A. Habich, M. Janowski, Z. Adamiak, P. Holak, H. Matyjasik, J.W. Bulte, W. Maksymowicz, P. Walczak, PLoS One 8(9), e74658 (2013). https://doi.org/10.1371/journ al.pone.0074658

35. S. Healy, J. McMahon, U. Fitzgerald, Neural Regen. Res. 13(9), 1520-1523 (2018). https://doi. org/10.4103/1673-5374.235222

36. M. Sezgin, B. Sankur, J. Electron. Imaging 13, 146-165 (2004). https://doi.org/10.1117/1.1631315

37. C.W. Chen, J. Luo, K.J. Parker, IEEE Trans. Image Process. 7(12), 1673-1683 (1998). https://doi. org/10.1109/83.730379

38. R. Kalicka, S. Lipiński, Biocybern. Biomed. Eng. 30(2), 15-27 (2010)

39. R. Kalicka, S. Lipiński, M. Browarczyk, Biomed. Technol. 58, 79-86 (2013). https://doi. org/10.1515/bmt-2012-0091

40. Y. Kimori, J. Synchrotron Radiat. 20, 848-853 (2013). https://doi.org/10.1107/S0909049513020761 
41. E. Smistad, T.L. Falch, M. Bozorgi, A.C. Elster, F. Lindseth, Med. Image Anal. 20(1), 1-18 (2015). https://doi.org/10.1016/j.media.2014.10.012

42. R. Tadeusiewicz, P. Korohoda, Computer Analysis and Image Processing, 1st edn. (Progress of Telecommunication Foundation Publishing House, Cracow, 1997), pp. 205-227. (83-86476-15-X)

43. E.R. Dougherty, R.A. Lotufo, Hands-on Morphological Image Processing, 1st edn. (SPIE Press, Washington, 2003), pp. 4-30. (978-0819447203)

44. H. Wang, Y. Zhou, B. Huang, L.T. Liu, M.H. Liu, J. Wang, C.Q. Li, Z.F. Zhang, T.W. Chu, C.J. Xiong, Tissue Eng. Part A 20, 908-920 (2013). https://doi.org/10.1089/ten.TEA.2012.0703

45. A.L. Goldberg, S.M. Kershah, J. Spinal Cord Med. 33(2), 105-116 (2010)

46. D. Jagannathan, V. Indiran, F. Hithaya, M. Alamelu, S. Padmanaban, Asian Spine J. 11(3), 365-379 (2017). https://doi.org/10.4184/asj.2017.11.3.365

Publisher's Note Springer Nature remains neutral with regard to jurisdictional claims in published maps and institutional affiliations.

\section{Affiliations}

\section{Seweryn Lipiński ${ }^{1}$ (D) Katarzyna Jezierska-Woźniak ${ }^{2,3} \cdot$ Aleksandra Habich $^{2,3}$. Joanna Wojtkiewicz ${ }^{4} \cdot K_{\text {Kamila Milewska }}^{2} \cdot$ Piotr Holak $^{5}$. Wojciech Maksymowicz ${ }^{2} \cdot$ Monika Barczewska²}

1 Department of Electrical Engineering, Power Engineering, Electronics and Automation, University of Warmia and Mazury in Olsztyn, Olsztyn, Poland

2 Department of Neurosurgery, Faculty of Medical Sciences, University of Warmia and Mazury in Olsztyn, Olsztyn, Poland

3 Laboratory for Regenerative Medicine, Faculty of Medical Sciences, University of Warmia and Mazury in Olsztyn, Olsztyn, Poland

4 Department of Pathophysiology, Faculty of Medical Sciences, University of Warmia and Mazury in Olsztyn, Olsztyn, Poland

5 Department of Surgery and Radiology, Faculty of Veterinary Medicine, University of Warmia and Mazury in Olsztyn, Olsztyn, Poland 